\title{
Access Grid Nodes in Field Research
}

\author{
by Nigel Fielding and Maria Macintyre \\ University of Surrey
}

Sociological Research Online, Volume 11, Issue 2,

< http://unw. socresonline.org.uk/11/2/fielding.html>

doi:10.5153/sro. 1385

Received: 8 Mar 2006 Accepted: 7 Jun 2006 Published: 30 Jun 2006

\begin{abstract}
This article reports fieldwork with an Access Grid Node ('AGN') device, analogous to video teleconferencing but based on grid computational technology. The device enables research respondents to be interviewed at remote sites, with potential savings in travelling to conduct fieldwork. Practical, methodological and analytic aspects of the experimental fieldwork are reported. Findings include some distinctive features of AGN interviews relative to co-present interviews; overall, there were some benefits and some disadvantages to communication. The article concludes that this new research interview mode shows potential, particularly once the difficulties associated with a new research technology are resolved.
\end{abstract}

\section{Keywords: Social Research Methods, Interview Methods, New Technologies for Social Research, Access Grid Nodes, Interview Communication, Witnesses at Court}

\section{What is an AGN?}

1.1 Advances in computing have recently enabled the development of greatly-enhanced computing power by linking together large numbers of computers in a way analogous to an electricity power grid. 'Grid' and High Performance Computing take three forms - the Data Grid, the Computational Grid, and the Access Grid ('AG'). Regarding the latter, a device called an Access Grid Node (AGN) enables projected images and sound to be exchanged in real time between different computers. The technology can be thought of as an enhanced form of video teleconferencing, with cameras and microphones at each AGN site relaying images and utterances of the people at that site to other AGN sites. There is no theoretical limit to how many sites can be linked, and participant numbers at each site are limited only by room size and technical configuration. While numbers of installations are increasing rapidly, they are chiefly used at present in the scientific community. Most are sited in universities.

1.2 To make communication as similar as possible to co-presence, AGNs deliver large format images (e.g., the current speaker in a meeting) to a projection wall and provide high fidelity sound. Computer displays, e.g. Powerpoint ${ }^{\mathrm{TM}}$ slides and software output, can be shown alongside the images of people, so all can see common objects of discussion. Up to 50 separate displays can be simultaneously shown. There is no lag of the sort associated with video teleconferencing. AGNs provide collaborative, computational and visualisation resources, with paralinguistic cues resulting from the large display format enabling more natural interaction. These qualities make AGNs a promising medium for activities requiring collaboration across distance. Their value for scientific 'meetings' in international collaborations was immediately realised but many activities normally requiring travel are in frame, including presenting classes and seminars without participants having to travel to a common site. This article explores another possible use, to conduct social scientific fieldwork.

\section{Fieldwork applications: the concept}

1.3 To the authors' knowledge, using AGNs to collect field data has not been tried before. In conventional interview or group discussion ('focus group') fieldwork, researchers often have to travel to participants or have participants assemble at some central point from a number of locations. With AGNs the researcher can stay put and the participants travel to an AGN local to them. Our research evaluates this form of 'virtual fieldwork'. It also documents the nature of AGN-mediated interaction and communication, by analysing and evaluating recordings of virtual fieldwork sessions. 
1.4 Qualitative evaluation is well-suited to testing AGNs as a fieldwork medium, as exploration of uncharted territory often involves qualitative methods. We have evaluated the use of AGNs for primary data collection by field experiments with a substantive focus on lay participants in the criminal trial process (the reason for this focus is discussed below). These experiments explore the use of AGNs to conduct nonstandardised interviews and moderator-led group discussions, systematically varying key elements (e.g., number of participants, number of sites). The principal emphasis is on communicative features - facilitators and inhibitors in AGN-mediated interaction, including technical hitches and the significance of visual cues noted in video-teleconferencing (Korschen, Pohl et al. 2002).

1.5 The selected fieldwork modes were chosen for their widespread use in contemporary social research. Moreover, interviews and group discussions are generic methods used both in quantitative and qualitative research. AGNs potentially offer a cost-efficient means of accessing dispersed, remote and hard-to-reach research populations; an example application might be in comparative research on users of a government service where the experiences of users in different regions are sought. Another application may be to elite interviews, where AGNs may make it possible to involve more senior research participants and research users. The methodology literature indicates that there is difficulty in obtaining participation of elite respondents in group discussions. An AGN being a sophisticated and novel medium, respondents such as judges, politicians and business executives may be attracted to participate in AGN fieldwork when they would decline a more conventional format. The AGN mode might enable, for example, a US and British judge to participate in a real-time group discussion, when it would be unlikely that both could be convened at a common location. The ability to display stimulus materials, such as a court transcript, is a further potentially helpful feature. Against such advantages must be weighed the necessary investment in facilities, and the cost of respondents attending at an AGN site; if one is nearby this may be modest, but respondents cannot be expected to commit themselves to more than local travel. However, both installation and respondent travel costs are likely to decline as the technology spreads.

1.6 AGNs may add a significant enhancement to the toolkit of social researchers, with returns not only for fieldwork but for collaboration amongst fieldworkers. There appears to be a particular potential in respect of international comparative research, at least where barriers of language do not obtain, and in work with elite respondents.

\section{Evaluating AGN-mediated fieldwork}

\section{Research design}

2.1 AGN technology is new, and its exploitation for social science applications is only beginning. Initial explorations must be informed by the AGN's methodologically distinctive characteristics relative to conventional co-present field methods, and relative to familiar online methods (e.g., asynchronous online interviews, video teleconferencing). Unlike a telephone or webcam interview, an AGN delivers the full range of interactional cues, and supports the dynamic display of high quality data images that can be used as response stimuli during interviewing/group discussions. Examples include displaying background statistics relating to the topic of interest, a copy of the interview guide, or vignettes (including 'movie' clips). Follow-up studies could also be supported. After a conventional initial stay, researchers could periodically 're-visit' informants via $A G N$.

2.2 The current research design systematically varies key features such as fieldwork mode (interviews, group discussion); number of AGN sites; number of participants at each site; presence/absence of local host; use/non-use of stimulus materials. Fieldwork has been conducted with a sample of social science, and computing science, students in four UK universities.

2.3 A later stage of the project will evaluate the utility of AGNs in fieldwork with elite respondents, namely, criminal trial judges in the UK and US. This focus relates to current research conducted by Fielding (2003; 2006). The fieldwork reported in the present article involves the same substantive focus, as a vehicle for the methodological experiments. Survey research suggests that up to $70 \%$ of lay participants are broadly satisfied with their experience at court (Whitehead 2001) but qualitative field research reveals that many crown court witnesses experience anxiety. Interviews and group discussions with the present respondents concerned attitudes to criminal justice, to give the fieldwork a substantive focus prior to questions relating to the fieldwork medium itself.

2.4 As an early set of experiments in the fieldwork use of AGNs, sample sizes are modest. Interactional features of fieldwork episodes are often studied for methodological purposes by relatively small-sample experiments, a contemporary example being Gobo's work (2001) on the effects of the survey introduction (the first few rounds of utterances) on response in standardised survey interviews, where conversationanalytic techniques were used to gauge the effects of different rapport-building devices. Small-sample 
qualitative work has long been method-of-choice for such exploratory work. While modest sample sizes are acceptable in exploratory research, the present number of AGN installations means that the sample universe is constrained in any case. The present distribution means that installations are both spatially and socially limited, but the availability of this methodological option is becoming more widespread, with a growth from about 220 AGNs in 2004 to over 550 in 2005.

\section{Technical considerations}

2.5 While technical aspects of work with AGNs are not the focus of this article, a comment is needed about recording. AGNs with inSORS software can record AG sessions using a feature called IG Recorder TM. This is supported by the AG Support Centre (AGSC) at Manchester Computing. AGSC store the recordings on their server and provide the service for a nominal fee for non-inSORS AGNs

(http://www.agsc.ja.net/services/igrecorder.php). This enables playback on request. All the video and audio streams from every site involved are available. However, these recordings are only accessible for a limited time, and may be accessible to others not involved in the fieldwork (see below for discussion of ethical matters). These are not criticisms of the AGSC, but the arrangements for researchers can be somewhat unwieldy. Moreover, output from IG Recorder cannot directly be manipulated as one might a VCR recorder. A tool called Arena is being developed that offers playback, fast forward and rewind, but output cannot be divided into segments in order to compile collections of similar features (as per the code-and-retrieve procedure characteristic of much qualitative data analysis; Fielding and Lee 1991).

2.6 The full functionality of AGNs is achievable in multicast mode. Multicast networking is the preferred mode for AGNs as the amount of data sent over the network is reduced, thereby decreasing the bandwidth and processing power required (Daw 2005). In many institutions, network firewall control issues limit multicast availability, and as a consequence such institutions are likely to operate in unicast mode. We mention this because much of the software supporting $A G$ sessions such as recording tools requires multicast networking. However, another software tool, AG-VCR, offers VCR-type functionality and can potentially be used in both unicast and multicast modes, but could only be used for a minority of our interviews. Consequently, all interviews were recorded using a conventional digital handycam and AG-VCR or Arena where possible. The video data from both recording modes was rendered into a format suitable for analysis using qualitative software but the data discussed here were analysed using Excel spreadsheets to segment extracts and compile these into collections of related features.

\section{Ethical considerations}

2.7 Ethical considerations are distinctive in AGN fieldwork. One must allow for the possible presence of third parties - technical operators - and agreements are needed with bodies such as the AG Support Centre if software is used that involves recordings residing elsewhere than one's own institution. Regarding the former, the necessary skills to operate AGNs during fieldwork sessions are readily acquired, enabling researchers to work without technical assistance, but this cannot be assumed of remote participants. Consent forms must be designed with this in mind. Regarding remote sites holding recordings, a written consent undertaking is required, or encrypting the session. Using a non-encrypted virtual venue can lead to being interrupted or overheard by others. However, being interrupted or overheard also occurs in conventional co-present fieldwork.

\section{The fieldwork}

2.8 Fieldwork has so far involved individual interviews. An 'information pack' was sent to all respondents containing details about AGNs, the research, and the interview. We experimented with the ability of AGNs to support dynamic display of graphs, text or streamed video by using static and interactive displays of the interview guide. The interview guide took a non-standardised format, with latitude for the interviewer to phrase probes in relation to main questions according to the displayed understanding of the respondent and taking into account comments the respondent volunteers that address matters the interviewer wished to discuss but had not yet raised. There is an initial interview explanation, when the consent form is completed if not already done when negotiating participation. The explanation notes that there are questions on a substantive research topic in criminal justice, and that questions will then be asked about the experience of being interviewed via AGN. After the interview the respondents were asked to complete an online 'demographic' form with questions about the respondent's gender, age and education. While this could be completed during the AG session it was more time efficient to complete it afterwards. Respondents were also asked about their use of computers and virtual communication (online message boards, video conferencing and video phones), and their experience of participating in qualitative research. 
as a research medium. Probes included questions relating to audio-visual quality. Respondents were asked if the technology was distracting, and to compare the AGN interview with a co-present interview and whether, given the choice, they would prefer a co-present or AGN interview.

\section{Sample}

2.10 A student sample was sought for convenience of recruitment. University students represent a large, easily-accessed group. Moreover, the majority of AGNs are located within universities, making a student sample a sensible choice for pilot work. The majority of respondents were postgraduates studying a range of social sciences, and computing. There were 14 student respondents, from four universities (University 1 $n=7$, University $2 n=4$, University $3 n=1$, University $4 n=2$ ). Half of the interviews were conducted AGN to AGN with the respondents using an AGN at their location and the researcher using the AGN at the University of Surrey. The other half of the interviews were conducted AGN to PIG (Personal Interface to the Grid; a PC with AGN software) where respondents used an AGN and the researcher used a PIG. For seven respondents the interview was the first time they had seen the researcher. Whilst node operators often greeted respondents, none were present during actual interviews.

2.11 Eight respondents were aged between 21 - 30 years, five between 31 - 40 and one between 51 - 60 . There were six female and eight male respondents. Five respondents were international students, from India, Nigeria and Ireland. For three respondents their first language was not English. Over half of the respondents had previous experience as qualitative research respondents $(n=8$, comprising observation $=1$, interviews $n=3$, group discussions $n=4$ ). Two of these instances were conducted by computer.

2.12 All respondents felt competent with computers, rating themselves between 6 and 10 on a confidence rating from $1-10$ (10 being the highest). Nearly half gave themselves a rating of $9(n=6)$. As an indication of familiarity with virtual communication we asked respondents whether they had used video conferencing, AGNs, online message boards and mobile phones (see table 1).

Table 1: Familiarity with virtual communication

\begin{tabular}{|c|c|c|c|c|}
\hline $\begin{array}{l}\text { Virtual } \\
\text { communication }\end{array}$ & $\begin{array}{l}\text { Video } \\
\text { conferencing }\end{array}$ & AGNs & $\begin{array}{l}\text { Online } \\
\text { forums }\end{array}$ & $\begin{array}{l}\text { Mobile } \\
\text { Telephones }\end{array}$ \\
\hline $\mathrm{N}$ & 5 & 4 & 10 & 14 \\
\hline
\end{tabular}

2.13 Our respondents were familiar with a range of virtual communication mediums. All used mobile phones, and text messaging was familiar $(n=13)$. In contrast, none had used video phones to make video messages. Nearly all respondents used online text-based communication ranging from email discussion lists to online message boards. While textual virtual communication was familiar to all, communication using video was less so.

\section{Communication in AGN-mediated interviews}

3.1 Virtual methods have considerable attractions for social and market research. Virtual interviews are commonly cited as traversing temporal and geographical boundaries. The internet can reach respondents who might be inhibited by offline research such as those suffering from debilitating conditions and those who prefer the anonymity offered by virtual communication. However, many existing evaluations have focused on text-based synchronous or asynchronous methods for interviewing, such as email or real-time chat rooms. Such interviews require, inter alia, computer literacy and typing skills. Less attention has been afforded to camera-based virtual interviews through mediums like webcams and video conferencing. Camera-mediated research potentially fosters interaction that may be nearer to co-present interviews than text-based media. The lack of spontaneity in asynchronous interviews is overcome in virtual synchronous environments and the addition of visual stimuli can add to the richness of the co-produced interaction. However, for some respondents and research topics the anonymity of text-based interviews may be preferred.

3.2 The use of computer-mediated communication for qualitative research interviews has been met with caution. Concerns relative to face to face methodologies include the displacement of time and space, creating 'dis-location' (Bampton and Cowton 2002) between respondents and researchers. This is the other side of the ability to traverse time, spatial boundaries and associated costs, that make virtual communication attractive, with the promise of making a myriad of communities accessible without leaving the office (Hine 2000). Virtual methods force researchers to re-appraise the notion of 'field' research; getting the 'seat of our pants dirty' is no longer a pre-requisite (LeCompte 2002). 
3.3 The changing definition of 'field' and the 'dis-location' between respondent and researcher are critical in understanding the nature of remote communication in research applications. The lack of co-presence has ethical implications (Allen 1996; King 1996) but here we focus on the development of the rapport regarded as critical for effective interviewing (Arksey and Knight 1999). How this is established without co-presence is fundamental in understanding virtual communication in general and in the development of effective virtual interviewing (Mann and Stewart 2000). We consider that virtual qualitative interviewing encompasses any interview conducted remotely, including several 'online' modes but also telephone interviewing.

3.4 Rutter (2002) highlighted the nebulous nature of the researcher's presence in text-based CMC, seeing a contrast between 'being there' and 'being absent'. The disembodiment inherent in text-based environments, lack of visual and aural communication, and physical distance, all may impact on social presence. But practitioners of text-based interviews have demonstrated that rapport can be developed online. To overcome disembodiment and lack of co-presence, users employ various devices to convey personality and tone, such as abbreviations, emoticons, capitalisation and punctuation. In text-based environments selfdisclosure has also been used to develop rapport and trust. Prior to real-time focus groups, O'Connor and Madge (2001) used a website with their photographs and biographies to disclose information about themselves to respondents. Personal information was also given in the focus group and elicited mutual disclosure by respondents. This method facilitated non-hierarchical relationships between themselves and their respondents, and promoted rapport between respondents. Kivits (2005) also found that reassurance from herself was fundamental and reinforced her online presence. Researchers often mirror verbal and body language to maintain positive relationships with respondents. In the absence of visual cues in text-based environments this has to be achieved by the content and style of messages. Kivits (2005) found that this had to be adapted to each individual. In asynchronous interviewing rapport is built over repeated interactions with respondents, as questions and probes are sent in successive exchanges (Bampton and Cowton 2002). Where questions are simply sent together with minimal probes, rapport and 'presence' are less likely to be achieved (Folkman Curasi 2001).

3.5 Whilst lack of visual and aural cues may impair communication in text-based CMC, the lack of visual identity can also have benefits. The veil of anonymity may be useful when researching sensitive topics, embarrassment being reduced by lack of physical presence (Campbell, Meier et al. 2001; Bampton and Cowton 2002), and encourage participation by those who may be excluded in face to face research, such as shy respondents, those with debilitating conditions, and those concerned with their physical appearance (Shaw, McTavish et al. 2000). Campbell et al. (2001) compared face to face and online focus groups.

Information gained was similar but respondents in online groups appeared to be more comfortable, so much so that some discussed personal health issues.

3.6 There are many parallels between debates concerning internet methodologies and those concerning telephone interviewing (Ibsen and Ballweg 1974). Anonymity afforded by lack of visual cues was also found useful when telephone interviewing about sensitive topics (Marin and Marin VanOss 1989) but appears to vary by ethnicity (Aquilino 1994). As in text-based interviewing, telephone interviewers utilise devices to foster rapport applicable to that medium:

'Verbal cues had considerable value in maintaining rapport. These cues included using common courtesies, personal titles, a cheerful and interested tone of voice, and the use of positive phrases. Careful timing of pauses and use of probes, coupled with affirming remarks, helped to maintain rapport without jeopardizing the integrity of the data.' (Davis Kirsch and Brandt 2002:80)

3.7 Whilst rapport can be effectively developed and rich data co-produced in text-based and telephone interviews, some topics/populations may require visual cues to facilitate interaction, analysis, or both. For example, televideo conferencing is increasingly being explored in the mental health field (Mclaren 2003), where the client/ health professional relationship is critical, and as a medium for employment interviews (Chapman, Uggerslev et al. 2003). However, Heath and Luff (1991: 102) highlight asymmetries in video interaction that render it a 'curious hybrid of face to face interaction and talk on the telephone' and Patrick (2001) notes several interactional challenges including eye contact asymmetries. It may be that AGNs come closer to co-present interaction than previous technologies; our results suggest that, at minimum, rapport was achieved and interviews proceeded to their conclusion. AG mediated fieldwork potentially enhances the depth of communication in virtual interviews.

3.8 Work on social aspects of technology has turned toward what users can do rather than what computers can do - a 'user focus'. Schneiderman (2003) emphasises the trend to 'universal usability' as a key transformation, where novices, the disabled, and the elderly are increasingly as likely to use computer technology as professionals, the able-bodied, and the young. Latterly attention has turned to how users shape technology from design to implementation (Oudshoorn and Pinch 2005). Users consume, modify, domesticate, design, reconfigure and resist technological development. Indeed, a primary theme of 
anthropological approaches to technology is the obstinacy of 'indigenous knowledge', the way that established modes of thought, practice and culture are applied to the new, and the processes by which the new is re-construed in terms of the established. Of course, it is not only a matter of what users do to technology but what technology does to users. Resistance and non-use are important in improving technology. The present relevance of user focus is the need for sensitivity to respondents' experience. It is already apparent that interview response can be collected via an AGN, but the key consideration is how respondents experience it.

3.9 The main concept in our analysis of communication features is that of 'engagement'. This means being engaged in the communication and can be seen as a continuum from being fully engaged to being disengaged. Being engaged was typified by not being aware of the lack of co-presence. In contrast, disengagement can be described as being overly conscious of the environment and the distance between respondent and researcher. Below we discuss barriers and facilitators to engagement. The unfamiliarity of the AGN environment was a cause for concern for some respondents. Moreover, the cameras, and display of the respondent's own image, created an initial artificiality, with elements of performance and stage fright apparent. Efforts were made to develop rapport, and this was generally successful. Indicators of rapport such as humour, self-disclosure and discussion of sensitive issues were evident. Moreover some respondents volunteered that rapport had been established. Where rapport was not felt to have developed this was attributed to a paucity of visual or paralinguistic cues. Positively, respondents felt they had more control over the interview and some were sufficiently confident and relaxed to break certain conventions of face-to-face interaction. Despite concerns about the environment and being under 'surveillance' many respondents felt they had formed a positive relationship with the researcher. Moreover, most experienced full engagement with the interview discussion. These respondents were only reminded of the lack of physicality when proceedings were disrupted, for example, by technical problems.

3.10 The video clips and textual extracts used below to illustrate elements of communication are annotated with numerical ID labels for individual respondents, with R01 the first respondent.

\section{Performance and camera awareness}

3.11 Respondents reported feeling somewhat apprehensive about the interviews. Their primary concern was the unfamiliar environment of the AGN, which dominated initial impressions. Most had no frame of reference for AG communication. The video wall, cameras, and other equipment indicated a technological environment; one respondent said this could be intimating and 'a bit Star Trek-ish', especially for respondents without experience of computers and technology (R07, male, computer confidence 9). First impressions were dominated by awareness of the cameras. Being the subject of four AGN cameras (and in some instances a handycam) was quite daunting despite respondents' confidence with computers and virtual communication.

3.12 The presence of cameras conveyed overtones of performance and some initially felt stage fright. Others responded by self-consciously fulfilling the role of a 'good' respondent and playing to camera as well as responding to the interviewer. Where individuals felt strongly self-conscious and under scrutiny, this may affect content and extent of response. When respondents could see themselves on the video wall they often reported consciously presenting themselves in specific ways, such as being attentive. One respondent highlighted the need to constantly demonstrate his attentiveness, such as looking at the researcher's video image even when, in face to face interaction, he felt it would be acceptable to look away. The display of attentiveness was also indexed by change of body language during the second half of the interview. In the substantive first half, respondents sat upright and mostly directly facing the video wall. During the de-brief both the respondent's and researcher's body language relaxed. Such transitions often occur when the formal business of an interview is completed, with an informal demeanour and more relaxed conversation before departure. However, respondents felt that the lack of co-presence had more of an impact on their contribution to the interview than concerns about self presentation (see "breaking the rules" and "communication and co-presence" below).

3.13 The technology and the cameras thus heightened the sense of a projected image of the self. As well as creating an additional layer of performance in the interview setting this may also have effects on self presentation and body image. Some respondents reported that they were conscious of their self image and being in this environment heightened such feelings. Although such concerns are not unique to AG communication, cues designed to give reassurance relative to co-present communication should be considered when designing AG fieldwork and cameras should be as unobtrusive as possible, a point respondents also made. One suggested that this would be more important for samples less familiar with computers and virtual communication. 
3.14 Despite the issues associated with the technology, particularly the ubiquity of the cameras, the respondents and researcher established rapport in most interviews. Moreover, the lack of physicality facilitated a realignment of the power imbalance between the researcher and the respondent that is often associated with fieldwork. Respondents' reported feeling happy to engage with the researcher remotely and demonstrated their ease at doing so during the interview by discussing sensitive issues. Some reported feeling in control of the interaction and the content of the discussion. On present evidence, the unfamiliarity of the AGN setting can increase respondents' apprehension and therefore impede the development and maintenance of an effective respondent/researcher relationship if strategies are not put in place to accommodate it. However, the respondents soon became at ease with the situation and consciously broke communication practices associated with face to face communication.

\section{Sensitive issues}

3.15 An indication that the AGN medium did not unduly obstruct rapport is that respondents felt able to discuss their unwillingness to appear as witnesses in court. One shared his experience of witnessing a violent crime and concern about being an eyewitness. This respondent was from India, and had witnessed a revenge murder. He was reluctant to be a witness because he was concerned for his safety. At the time he was single, and he elaborated that his concerns would be greater if he had a family. Fortunately the perpetrator came forward and confessed. However, the respondent's concerns over safety were so great that if this had not happened he still would not have been willing to testify. This particular respondent's viewpoint breaches socially desirable responses in the UK, unlike within a culture where revenge crimes are commonplace and where, as the respondent explained, citizens often know the identities of perpetrators. The example illustrates that despite interacting remotely with a stranger, respondents felt able to discuss sensitive issues.

\section{Breaking the rules}

4.1 A number of respondents reported a sense of being in control of the interaction and of how much or how little they said. They also felt able to break some conventions of face to face communication, such as by often breaking 'eye contact' and looking away from the researcher. This often occurred when respondents were thinking through their responses; there was testimony that this allowed more time to reflect on responses. Additionally, one respondent felt able to make notes during the interview about what she wanted to say in the de-brief, whilst another respondent asked the researcher to take off her glasses to see whether this had an impact on making eye contact. These actions highlight that the respondents felt empowered to break face to face communication conventions whilst also demonstrating that they were actively engaged with the interview rather than passively responding. Respondents attributed this to the lack of physicality, that instead of engaging with a person in the same location they were engaging with an image on the video wall.

4.2 The following clip highlights how the lack of physicality can impact on interaction. The researcher asks the respondent to compare her AGN experience to that of face to face communication. The respondent explains that the removal of the physical presence facilitates interaction that is more relaxed than face to face communication. Whilst responding to the researcher's question she pauses and breaks eye contact by looking to her left. She used this strategy throughout the interview. In this clip she explains that interacting with an image enables her to break some conventions of face to face communication and that this allows her to think through responses before verbalising them. The lack of physicality can increase the respondent's control and power over the interview. Furthermore despite initial concerns over the unfamiliarity of the setting, interacting remotely can foster a comfortable and relaxed research setting, such that for some respondents this was preferable when meeting a 'stranger' like a researcher (see Appendix 1 for transcript of the clip).

Video clip 1: Breaking the rules (R10, female, Computer confidence 9)

\section{Communication and co-presence}

5.1 Whilst ensuring respondents are not disempowered by the research process is critical, it is also important that response be accurate. One respondent reported that because she felt in control of the interview and her contribution she also felt she could be 'evasive'. This suggests the need for balance between not feeling pressurised to talk about things that are uncomfortable to discuss and responding in a way that might be misleading. Once again this was attributed to being in a different location to the researcher.

5.2 Another issue that respondents raised was the length of silences. Some felt that they talked excessively in the interview. This was attributed to wanting to fill in any silences, rather than taking time to 
reflect. Respondents commented that they repeated themselves in an effort to fill in these silences and to ensure that they expressed the message that they wanted to convey. While also featuring in conventional fieldwork, there is still some lack of paralinguistic cues in AGN work that may influence this feature. Clarity of facial expression such as eye and mouth movement is one cue raised by respondents. In comparison to telephone interviews AG interviews enable respondent and researcher to see what the other is doing during the interview and thus monitor engagement. Respondents rated $A G$ interviews favourably in comparison to telephone interviews. Moreover, they classified AG communication as a 'middle ground' - closer to conventional interaction than telephone communication and video conferencing due to the immediacy of verbal and visual communication. However, they highlighted that some of the subtlety and detail of cues readily available face to face was still lacking. Respondents commented that in everyday conversation we look for turn-taking cues. Some of these were present, such as intonation and nodding, other more subtle cues such as hearing the other person take a breath in preparation for speech, and slight facial expressions, were less apparent. However, multicast functionality, experience with room layout, and discreet camera installation offer substantial means of addressing these issues.

\section{Engagement}

5.3 As noted earlier, respondents initially found the environment and the presence of cameras challenging. Yet despite concerns with being under constant camera surveillance the respondents were able to form a positive relationship with the researcher, such that they felt in control of the communication and at ease to discuss sensitive issues. One explanation for this is the concept of engagement. During the de-brief the respondents discussed being engaged with the researcher and the topic. When respondents felt engaged the lack of physicality was not a barrier to interaction. Those who felt engaged during the interview were happy to use the AGN to communicate.

5.4 One problem inherent in virtual communication is the lack of shared experience. Being remote means connecting in a shared virtual space whilst remaining in different environments. For most respondents during the interview the setting became less important as the interview proceeded and they became engaged with the substantive topic. One respondent explained that once you were in the 'guts of the interview' (R07, male, Computer Confidence 9) being in different locations (and therefore contexts) was not an issue, the discussion thereby being analogous to face to face interaction in the same room. This highlights the importance of engagement, both with the researcher and the topic. During periods of engagement the discussion rather than the lack of co-presence thus became the respondent's main focus of attention. However, being engaged in the interview could easily be disrupted, making the virtual nature of the communication apparent. The context, cameras, technical problems and disruptions all affected levels of engagement. For the respondent in the following two video clips a lack of shared physical space meant a lack of shared experience. During the interview someone else entered the researcher's room, causing the researcher to leave her seat and be out of view. The following clips highlight the lack of a shared context inherent in remote communication. In the first the researcher is disturbed and stops the interview to resolve the situation. During this time the respondent cannot hear or see what is going on off-camera.

Video Clip 2: Shared context, shared experience: Interruption (R04, female, computer confidence 9)

5.5 The disruption is quickly resolved and the respondent continues her response. Although the interruption only affected the flow of the interaction temporarily it did reiterate the lack of co-presence and thus shared context. During the de-brief the respondent reflects on the lack of a shared space and shared experience in terms of jointly resolving the disruption (see Appendix 2 for transcript).

Video Clip 3: Shared context, shared experience: Discussion of interruption (R04, female, computer confidence 9)

\section{Barriers to engagement}

5.6 The main barriers to engagement were the physical reminders that the respondents were communicating remotely. These were disruptions, the cameras, awareness of one's own image and technical problems.

5.7 Some respondents could see themselves on the video wall. This appeared to be something of a distraction. Very often this became a reminder of the context: "... sometimes I was distracted by my own image and so I would think 'what was I saying?' 'oh look at me I'm there' and I'd kind of lose my thread and I'd think hang on a minute, you're talking about the criminal justice system' (R09, female, Computer confidence 10). Movement often exacerbated this; "I keep getting distracted by my hand movements" (R01, female, Computer Confidence 8). This was troublesome as gesture was used to highlight or emphasise 
views or experience, indicating full engagement. This sign of engagement became an additional reminder of the context and remote nature of the interaction.

5.8 Further barriers to engagement were interruptions and technical problems. Whilst most ignored technical problems during the interview, raising them instead as a concern during the de-brief, one respondent perceived a disparity in the quality of audio and visual stimuli and found this distracting throughout. This affected full engagement because attention was focused on the difference between the video and audio, the former being poor relative to the latter. This made her perceive the communication as unnatural. There were technical problems in other interviews but analysis of recordings revealed that most respondents ignored technical hitches and focused on the interaction.

\section{Conclusion}

6.1 The concept of engagement provides analytic purchase in understanding research participants' response to AGN fieldwork. It highlights some communicative features distinctive of this medium and indicates there is a somewhat mixed picture with respect to how AGN-mediated interviews compare to conventional interviews and those conducted via video teleconferencing. Positive elements include the thorough record that researchers gain from digitally-recorded audio-visual data, the excitement that participants manifestly feel about participating in a technologically-sophisticated fieldwork environment, their feeling that they can control the interaction in ways that seemed less available in face-to-face interviews, and the saving in researcher travel time. Negative elements include an extra level of self-consciousness that is perhaps inevitable when one is aware one is 'on camera' as well as being audio-recorded, the issue relating to encryption noted earlier, and a series of technical glitches and consequent delays and interruptions. The technical matters are inevitable in a new research technology. A partial response to the self-consciousness issue would be to better conceal the cameras, but this will never entirely overcome the problem. However, most fieldworkers nowadays record interviews by some means and there is much testimony that self-consciousness fades as sessions proceed. With smoother technical performance this may be the case with the AGN, too.

6.2 It should also be noted that a student sample is a sample particularly knowledgeable about research methodology and, for most respondents, about technology as well. Respondents from the general public may not be so aware of some of the features our sample remarked upon, having little to compare it to other than interviews most people encounter, such as job interviews or medical consultations, both of which are somewhat nervewracking and to which the AGN interview may actually compare favourably.

6.3 Throughout this article we have highlighted the impact of the context on the interview process, particularly awareness of cameras and video recording. Recording of interviews frees researchers from extensive note-taking, allowing them to listen and interact with respondents. However, there will always be some concern over the impact of being recorded on what is said by respondents. We share Speer and Hutchby's (2003) position, that recording devices should be seen as part of the interaction and researchers must attend to how the respondents orient to being recorded and the role this plays in the interaction.

Instead of seeing the presence of a recording device as necessarily contaminating what would otherwise be a pristine occasion of real-world interaction, and rather than worrying over the extent to which participants' noticing of, making reference to or otherwise displaying orientations to the fact of their being recorded gets in the way of the 'authentic' talk we are interested in gaining access to, we argue that social scientists should investigate precisely what it is that participants are doing when they orient to being recorded: how might what they do in such orientations play a part in the ongoing construction of specific situation interactions?

(Speer and Hutchby 2003) p317

6.4 In the AGN setting, understanding how participants respond both to the cameras and to being recorded is critical to understanding AG-mediated interaction. This also applies to any other AG use, such as meetings and training events. In reference to its use for data collection purposes, exploring how respondents orient themselves to the setting is critical for identifying barriers and facilitators of communication.

6.5 This apart, our fieldwork produced, in respect of the substantive topic, the volume and richness one associates with qualitative data, and some apt and insightful evaluative comments on virtual fieldwork. The present experience cannot claim any more than that the medium shows promise as a potential new mode of the research interview, but that is sufficient in itself to demonstrate both the robustness of the interview as a form of human communication and the possibility that this new technology can contribute to the social researcher's toolkit. 


\section{Appendix}

The appendices contain the transcripts for video clips 1 and 2. In both cases two forward slashes indicate where there is overlap in speech.

\section{Appendix 1}

\section{Transcript for Video clip 1: Breaking the rules (R10, female, Computer confidence 9)}

MM: How do you think, were, were there that, you felt as if maybe we were in the same room talking? Or was it very much you felt you were there and I was here or

R10: Umm (pause)(looks to her left) Yeah well er I mean I suppose it doesn't feel like you're in Surrey, it does feels like you're just maybe at the top of the room like kind of (points to the end of the room where to the video wall). Err so like you are there. I think at the same time umm it's easier to kind of withdraw you know my eye gaze and that like you know if I'm thinking of a question I can kind of look away // MM: Yeah // R10: because you're just (laughs) // MM: Yes (laughs) // R10: a screen like you know (laughs) // MM: (laughs) // R10: (laughs) as opposed to normal conversation l'd have to keep up that eye contact // MM: Oh Okay // R04 And, And you know manage err manage the interaction a little more - yeah

MM: The the - that's quite interesting so you think as b, because its just screen you've got more freedom to maybe look away rather than

R10: Yeah - I think its something got to do with umm you know the rules about interaction MM: Yeah // R10: and like you know.

R10: So when you're with somebody // MM: Yeah // R10: You do have other cues you know like touch or whatever that you can use as well. Umm but I do feel that I can kind of break the rules of conversation sometimes and just look off (looks to her left to)(laughs)// MM: Oh Ok (laughs) // R10: kind of (laughs) // MM: (laughs) // R10: Yeah. So umm // MM: That's interesting // R10: And it would be easier to do that, yeah // MM: Umm

R10: it's not like the social reprisals are as

MM: Yeah // R10: prevalent possibly

MM: Ok and do you think there are other, other sort of rules that you've broken or umm can you think of anything? //R10: Umm (pause)(looks down) maybe l've used longer silences than I would usually // MM: ok

R10: yeah thinking of things - possibly

MM: yeah ok that's g, I mean do you think // R10: Only slightly// MM: yeah // R10: but // MM: yeah, yeah

\section{Appendix 2}

Transcript for Video Clip 3: Shared context, shared experience: Discussion of interruption (R04, female, computer confidence 9)

R04 Probably.

MM right

R04 It's probably, I think it i, i, i, it to me it would always, in an interview setting it would always be better to have, be in the same room as someone but it depends on why it was chosen and if that outweighs that then I could can with it completely

MM Why do you think umm being in the same room would be so much more preferable in the interview situation?

R04 Um that's what I'm much more familiar with. // MM Ok // Probably mostly. Umm. This is the first time I've done this // MM Yeah // so

R04 Umm (pause) I don't er, I mean don't know, I just, probably just because of familiarity really. And because we're sharing a space and therefore sharing a level of experience ie when you're in, when the door opens I, - we're both experiencing it // MM Umm // R04 And we both respond to it. And I'm not kind of left 'what's going on?' sort of thing, and it just, from that point of view. Umm 


\section{Useful link}

Our website provides non-technical information concerning AGNs and the practicalities of using AGNs in social research. It also provides links to other sites which provide information, support and training concerning all aspects of AGNs from installation to their effective use. http://www.accessgrid.surrey.ac.uk

\section{Acknowledgements}

This article reports work from the project 'New Technologies, New applications: Using AGNs for Social Research'. The research was funded by the Economic \& Social Research Council under award RES-14925-1005. We would like to thank our respondents for taking the time to share their views and experiences. We also very much appreciate those who have provided technical support at our own institution and at remote sites, and those at other institutions who have assisted us in gaining access to the sample respondents.

\section{References}

ALLEN, C. (1996). "What's wrong with the "Golden Rule"? Conundrums of conducting ethical research in cyberspace." Information Society 12(10).

AQUILINO, W., S (1994). "Interview Mode Effects in Surveys of Drug and Alcohol Use: A Field Experiment." The Public Opinion Quarterly 58(2): 210-240.

ARKSEY, H. and P. Knight (1999). Interviewing for Social Scientists. London, SAGE Publications.

BAMPTON, R. and C. Cowton, J (2002). "The E-Interview." Forum: Qualitative Social Research [On-line journal] 3(2).

CAMPBELL, M., K, A. Meier, et al. (2001). "Health Behavior Changes after Colon Cancer: A Comparison of Findings from Face-to-Face and On-Line Focus Groups." Family Community Health 21(3): 88-103.

CHAPMAN, D., S, K. Uggerslev, L, et al. (2003). "Applicant reactions to face-to-face and technologymediated interviews: a field investigation." Journal of Applied Psychology 88(5): 944-53.

DAVIS Kirsch, S., E and P. Brandt, A (2002). "Telephone Interviewing: A Method to Reach Fathers in Family Research." Journal of Family Nursing 8(1): 73-84.

DAW, M. (2005). "Advanced Collaboration with the Access Grid." Ariadne(42).

FIELDING, N. (2006). Courting Violence. Oxford, Clarendon.

FIELDING, N 'Executive summary: A Research Study of Procedures and Court User Experiences in Crown Court trials of offences of physical violence', Guildford: Institute of Social Research, pp 10.

FIELDING, N and R.M. Lee 'Computing for qualitative research: options, problems and potential', in N. Fielding and R. M. Lee, eds., Using computers in qualitative research, Sage 1991, 1-13.

FOLKMAN CURASI, C. (2001). "A critical exploration of face-to-face interviewing vs. computer-mediated interviewing." International Journal of Market Research 43(4): 361-375.

GOBO, G. (2001). "Best practices: rituals and rhetorical strategies in the 'initial telephone contact'." Forum Qualitative Sozialforschung 2(1).

HEATH, C. and P. Luff (1991). Disembodied conduct: Communication through video in a multi-media office environment. In Proc. of CHI'91, ACM.

HINE, C. (2000). Virtual Ethnography. London, Sage.

IBSEN, C., A and J. Ballweg, A (1974). "Telephone Interviews in Social Research: Some Methodological Considerations." Quality and Quantity 8: 181-192.

KING, S., A (1996). "Researching internet communities: proposed ethical guidelines for the reporting of results." The Information Society 12: 119-127. 
KIVITS, J. (2005). Online Interviewing and the Research Relationship. Virtual Methods: Issues in Social Research on the Internet. C. Hine. Oxford, Berg.

KORSCHEN, M., J. Pohl, et al. (2002). "New techniques in qualitative conversation analysis: computerbased transcription of video-conferences." Forum Qualitative Sozialforschung 3(2).

LECOMPTE, M. (2002). "The transformation of ethnographic practice: past and current challenges." Qualitative Research 2(3): 283-299.

MANN, C. and F. Stewart (2000). Internet Communication and Qualitative Research: A Handbook for Researching Online. London, Sage.

MARIN, G. and B. Marin VanOss (1989). "A Comparison of Three Interviewing Approaches for Studying Sensitive Topics with Hispanics." Hispanic Journal of Behavioral Sciences 11(4): 330-340.

MCLAREN, P. (2003). "Telemedicine and telecare: what can it offer mental health services?" Advances in Psychiatric Treatment 9: 54-61.

O'CONNOR, H. and C. Madge (2001). "Cyber-Mothers: Online synchronous interviewing using conferencing software." Sociological Research Online 5(4).

OUDSHOORN, N. and T. Pinch, Eds. (2005). How users matter. Cambridge MA, MIT Press.

PATRICK, E., J (2001). Barriers to Collaboration: User-Centred Research and the Access Grid, Motorola Labs Research Report.

RUTTER, J. (2002). Ethnographic Presence in Nebulous Settings: A Case Study. ESRC Virtual Methods seminar series, Brunel University.

SHAW, B., R, F. McTavish, et al. (2000). "Experiences of Women With Breast Cancer: exchanging social support over the CHESS computer network." Journal of Health Communication 5(2): 135-.

SPEER, S., A and I. Hutchby (2003). "From ethics to analytics: Aspects of participants' orientations to the presence and relevance of recording devices." Sociology 37(2): 315-337.

WHITEHEAD, E. (2001). Witness satisfaction: findings from the Witness Satisfaction Survey 2000.

London, Home Office. 